\title{
Biologic Waste: Consistent Performance with an
}

\section{Alternating Field}

\author{
Hans-Jürgen Huber \\ Industry Sales Development Chemicals, Siemens AG, Karlsruhe 76187, Germany
}

\begin{abstract}
Biomass plants often struggle to capture flow measurements reliably. High amounts of dry solids and fats complicate the measurement with an MID (Magnetic-Inductive) flowmeter and make it susceptible to faults. To overcome this impediment, the waste water treatment plant in Innsbruck, Austria, relies on electromagnetic pulsed AC (Alternating Current) flowmeters. Compared to pulsed DC (Direct Current) devices, AC devices are able to build up magnetic fields that are ten times stronger. Equipped with this capability, the Sitrans Transmag 2 is able to guarantee a constant and also high measuring accuracy, zero point stability and signal strength regardless of impurities in the medium or fluctuations in the magnetic field.
\end{abstract}

Key words: Biomass, wastewater treatment, process instrumentation, flow measurement, pulsed AC meter, pulsed DC meter, Transmag 2.

\section{Introduction}

Innsbruck's treatment plant IKB (Innsbrucker Kommunal Betriebe) AG ranges among the most sophisticated treatment facilities across Europe. Day after day, up to $165,000 \mathrm{~m}^{3}$ of wastewater coming from the city of Innsbruck and its 14 surrounding municipalities are being handled in a multistage process to biologically and mechanically clean the volume of water before it reenters the natural water cycle of the river Inn.

Additionally, the plant handles 8,000 tons of organic waste each year to produce biogas.

In view of a holistic use of all sources of energy, the biogas produced within the fermentation process is supplied to the block heat and power unit on site to generate electricity. This process generates $21 \mathrm{MWh}$ of electricity daily. A quantity which serves 1,600 4-person households to cover their daily demand. It is just as well that the co-generation unit re-uses its waste heat for another purpose: the heating of its own process, which is essential and does in turn increase

Correspongding author: Hans-Jürgen Huber, graduated engineer, field of activity: industry specialist process automation in chemical applications. the efficiency of the entire plant.

However, the actual process of turning biological waste into energy begins much earlier. Before the organic waste can be fed into the fermentation process, it requires extensive filtering to eliminate impurities such as plastics, rocks and metals. To obtain a purified medium, the biological waste is being ground and blended to a chewy mass together with process water and the delivered fats. This liquid mixture is being stored in bunkers before the remaining impurities are being separated and eliminated at a hydrocyclone. Only now can the treated and cleaned organic waste be partnered with the sewage sludge from the water treatment facilities and left to ferment in two digestion towers.

\section{Avoiding Impediments and Signal Failures}

Within these process steps, the measurement of flow rates is imperative. As biomass qualifies as an electrically conductive medium, the sensor of choice is an electromagnetic one. It is well suited for the measuring task and the most cost-effective solution at the same time. Magmeter devices apply a magnetic field to the metering tube, which contains the biomass and measure the induced voltage, which in turn 
provides information about flow speed. When then factoring in the diameter of the pipe, the flow rate can be precisely determined. Common mag meters work according to the principle of pulsed DC, which works well for media with a certain minimum conductivity and even in aqueous solutions. Biomass, however, does have its peculiarities!

For one thing, the high amount of contained fats (Fig. 1) can have an isolating effect on the measuring electrodes, reducing the induced potential and making it impossible to measure. The measuring performance of pulsed DC devices is also negatively affected, whenever bigger green cuttings scrape over the electrodes causing signal loss-process conditions, which should not even arise in the first place.

\section{Intelligent Measurement Principle for Reliable Results}

To make sure that the measuring signal is not momentarily interrupted by high levels of fats or other foreign media, the water treatment plant Innsbruck relies on electromagnetic pulsed AC flowmeters. While pulsed DC meters reach their limits whenever impurities are concerned, pulsed AC meters prove their capability even under harshest conditions. In a direct comparison, their advantage becomes evident: compared to pulsed DC devices, AC devices are able to build up magnetic fields ten times stronger. Equipped with this capability, the Transmag 2 is able to guarantee a constant and high measuring accuracy, zero point stability and signal strength regardless of impurities in the medium or fluctuations in the magnetic field. The Transmag 2 does hence combine the advantages of pulsed DC devices (high signal strength) with the advantages associated with pulsed AC devices (stable zero point). The Transmag 2 also has an answer to the fluctuations in the magnetic field, voltage or temperature, which usually take their toll on the measurement accuracy of DC devices: these influences are being cut out thanks to the PAC (Pulsed Alternated Current) Technology and an additional reference coil (Fig. 2). Even in applications with very low media conductivity as low as $0.1 \mu \mathrm{s} / \mathrm{cm}$ this setup permits reliable measurements. The treatment plant Innsbruck relies on this technology in a number of sub-processes and profits from further parametrization options. With the push of a single button, application-related disturbances can be suppressed. This task is being tackled by the slurry mode which —once individually parametrized — will use a tolerance band to increase the stability of the measurements (Fig. 3). Whenever measurements lie outside the set tolerance range, this reading will be compared to previous measurement readings within a defined blanking period and used to calculate an average value. By doing so, the device carries on working even if signal disturbances occur and retains

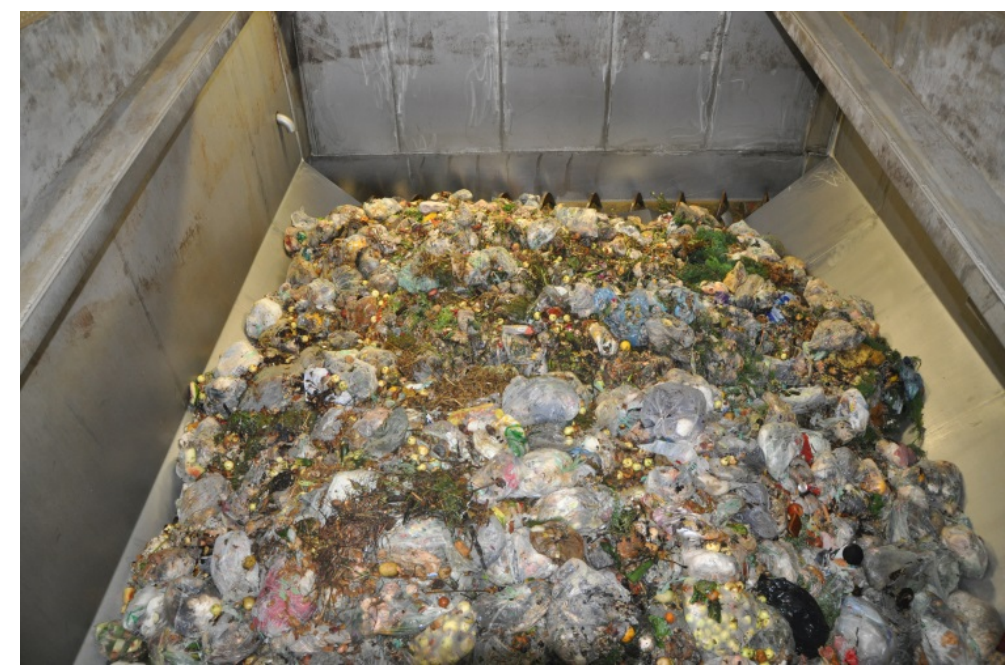

Fig. 1 Biological waste including impurities on the premises of Innsbrucker Kommunalbetriebe. 


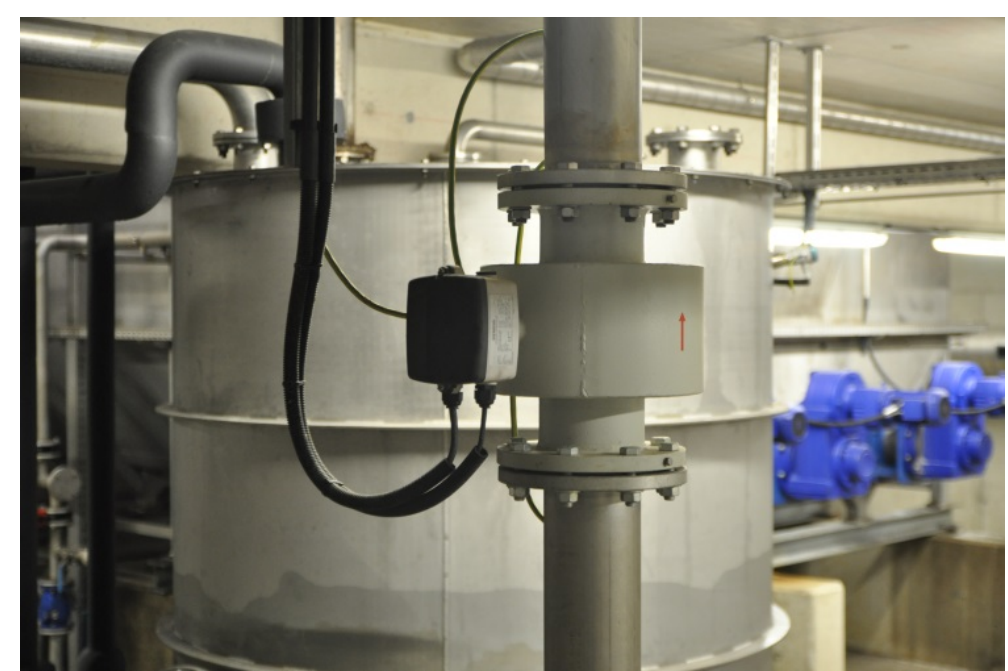

Fig. 2 Reliable even under harshest conditions and high concentrations of impurities: the Transmag 2 flowmeters in the IKB handle up to $40 \mathrm{~m}^{3}$ an hour.

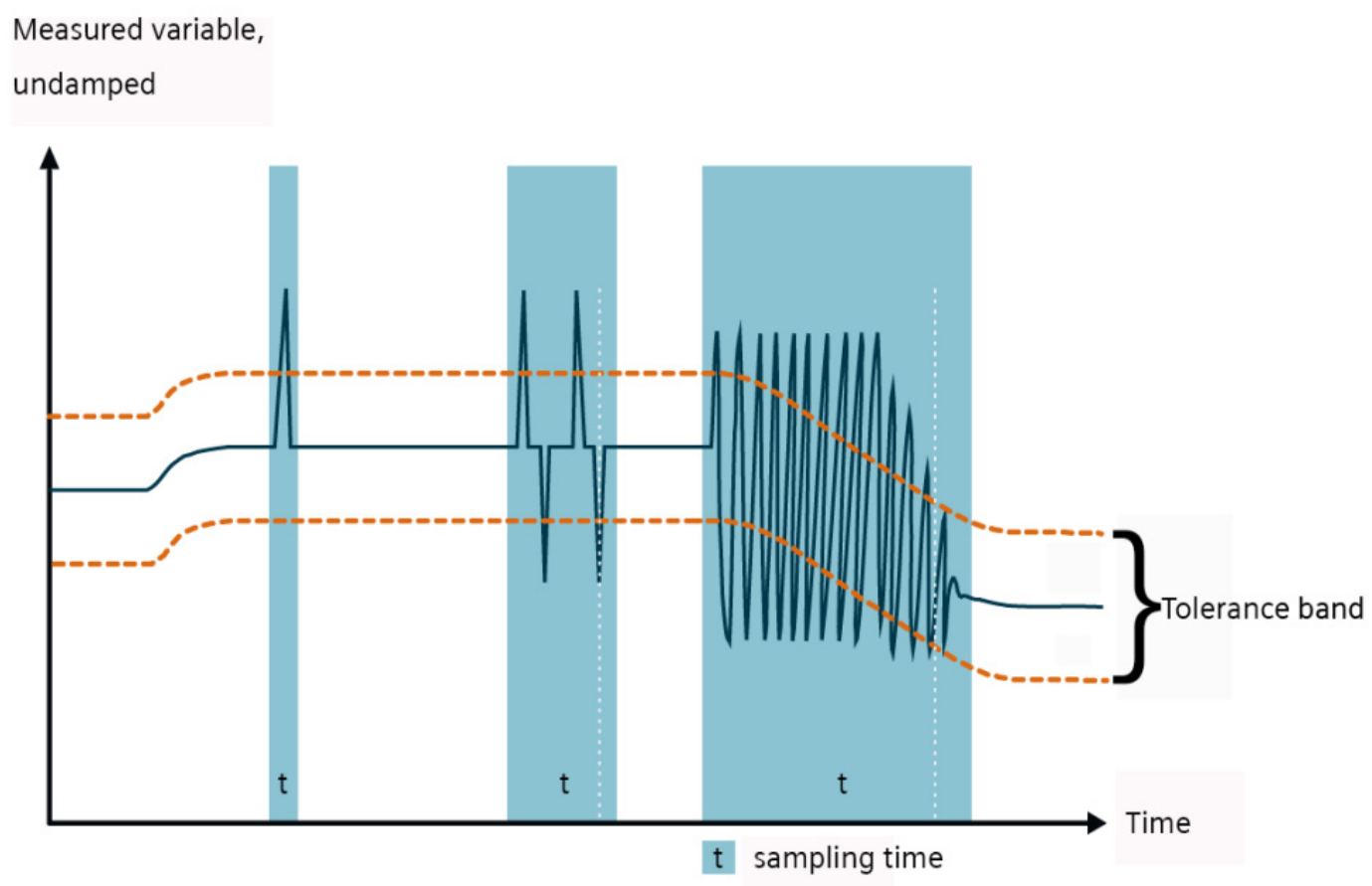

Fig. 3 Failure-free flow measurement: technical principle of the Slurry Mode integrated in Transmag 2 to improve the stability of the measured values.

the most recent measuring value. In cases where the disrupted signal is permanent, the slurry mode will continue to calculate average values until the deviation to the tolerance band is no longer present. Once this status is achieved, the device returns to normal operation. This mode is especially suited for media with entrapped air or high concentrations of solids, such as biomass.
Not only the quality of the measurement but also the quality of the device itself have been taken into consideration when developing the Transmag 2. Liner materials specifically designed for abrasive media and a selection of electrode materials ensure that wear and tear is reduced to a minimum ensuring quality measurements and a low cost of ownership due to longevity of operating life. The treatment plant's 
Transmag is lined with Novolak, a highly robust material with a smooth, hard surface. With this liner in place, the biomass can pass the measuring tube without considerable friction. In addition, this liner material protects the measuring tube against abrasion and corrosion. Even at high pressures and temperatures or under vacuum conditions Novolak does endure.

\section{Right Measurement Technology for Energy Efficiency}

Having the right measurement technology in place is beneficial also for superordinate processes and tasks. In the Innsbruck waste treatment plant, the performance of downstream pumps is being controlled and adjusted on the basis of the captured flow measurements. Especially when starting up the plant, when the flowmeters must handle up to $15 \%$ solid concentrations, precise measurements are vital to control downstream actuators as efficiently as possible. This supports the overall aim to attain maximum separation efficiency for the non-organic materials in order to exploit the full potential of the hydro cyclone: Because the lower the density difference between liquid and solids, the more efficient does the hydro cyclone work.

\section{Treatment Plant Integral Part of Smart Municipal Energy Use}

The extension of the IKB treatment plant to a district heating power station for surrounding businesses and the connection to Innsbruck's district heating system won the company the renowned EPCON award. The optimal and efficient operation of the plant is not coincidence. Among other things, it is rooted in a well-functioning, efficient measuring technology.

The Transmag 2 provides its reliable contribution and is well-equipped for higher demands in case of a further expansion of the facilities. High degrees solid shares and impurities in the medium do not interfere with its measuring performance.

\section{Conclusion}

For flow measurements in biomass with high shares of substrate, fats or green cuttings, an MID with an alternating field, like the Transmag 2, is the device of choice to consistently obtain reliable and meaningful measurement values. 